\title{
Determination of the expression of three fimbrial subunit proteins in cultured Trueperella pyogenes
}

Mengcheng Liu ${ }^{1,2 \dagger}$, Bing Wang ${ }^{1,2 \dagger}$, Hongmin Liang ${ }^{1,2}$, Bo Ma ${ }^{1,2}$, Junwei Wang ${ }^{1,2}$ and Wenlong Zhang ${ }^{1,2^{*}}$

\begin{abstract}
Background: Trueperella pyogenes is a commensal and a significant opportunistic pathogen in animals. A variety of identified or putative virulence factors are considered to significantly contribute to the occurrence of T. pyogenes infection in different species. However, these virulence factors are not fully understood.

Results: In the current study, the genes encoding putative fimbrial proteins, i.e. Fim A, Fim C, and Fim E, were cloned. Recombinant Fim A (rFim A), Fim C (rFim C), and Fim E (rFim E) were prepared and used to generate rabbit anti-rFim A, anti-rFim C, and anti-rFim E serum, respectively. Using these sera, we found that only Fim E was constitutively expressed in T. pyogenes. The expression level of Fim E in T. pyogenes peaked within 6-10 h of culture period in pH 7.5. Fim E protein expression was unaffected by anaerobic condition, but was inhibited by the microaerophilic condition. Tube agglutination tests indicated that Fim E was exhibited on the surface of T. pyogenes cells because anti-rFim E serum caused strong agglutination. Additionally, the blots for Fim A detection showed nonspecific reactions. Furthermore, the tube agglutination tests showed that anti-Fim A serum failed to cause agglutination of T. pyogenes cells, which indicated that Fim A was not, or poorly, expressed in cultured T. pyogenes. Anti-rFim C serum caused strong agglutination. However, the blots for Fim $\mathrm{C}$ detection showed a strong nonspecific reaction. Thus, the expression of Fim $C$ was difficult to be determined using the current method.
\end{abstract}

Conclusions: Fim E was expressed in cultured T. pyogenes. However, Fim A was either not or poorly expressed in cultured T. pyogenes. Moreover, Fim C expression was not determined using the current strategy.

Keywords: Expression, Fim A, Fim C and Fim E, Trueperella pyogenes, Virulence factors

\section{Background}

Trueperella pyogenes is a Gram-positive rod-shaped bacterium [1]. This organism is a commensal and a common opportunistic pathogen in different domestic and wild animals [2-4]. Moreover, human beings can be infected by this bacterium; however, the infections are generally related to occupational exposure [5].

The ability of the T. pyogenes in causing infections in different species could be, at least partially, attributed to

\footnotetext{
*Correspondence: zhangwenlong@neau.edu.cn

${ }^{\dagger}$ Mengcheng Liu and Bing Wang contributed equally to current study

${ }^{1}$ College of Veterinary Medicine, Northeast Agricultural University,

Changjiang Road 600, Harbin 150030, Heilongjiang, People's Republic

of China

Full list of author information is available at the end of the article
}

various virulence factors that are encoded in its genome [1]. The virulence factors of this bacterium include exotoxins (pyolysin), exoenzymes (proteases and DNase), and adhesins [1]. Among these virulence factors, components that mediate the adhesion of the organism to host cells play essential roles in establishing the infection $[6,7]$. For $T$. pyogenes, these host cell adhesive factors not only work in establishing the infection but also when the organism acts as a commensal on the various mucous membranes of the hosts. Thus, investigations have been conducted on adhesins to help understand the pathogenicity of T. pyogenes and its mechanism in terms of switching from a commensal to a pathogen. Many adhesive components, such as collagen-binding protein A, fibronectin-binding protein, and fimbriae, that might 
mediate the adhesion of this organism to the host tissue, have been reported [1]. The genes encoding these adhesive components have been extensively used as molecular markers for identifying, genotyping, or predicting the virulence of $T$. pyogenes $[3,8]$. However, evidence of adhesin expression in T. pyogenes cells is extremely limited [9-11]. T. pyogenes has been reported to express fimbriae [1]; however, the properties and constitution of this structure have not been studied. Putative fimbrial subunit proteins, such as Fim A, Fim B, Fim C, Fim E, and Fim J, have been reported to be expressed in T. pyogenes. However, only the genes, mRNAs, or the existence of specific antibodies against these proteins in T. pyogenes-infected animals have been detected $[1,12,13]$.

In the current study, we aimed to determine the expression of three putative fimbrial subunit proteins, namely, Fim A, Fim C, and Fim E, in cultured T. pyogenes. However, only the expression of Fim E was detected. These results will contribute to the work on molecular pathogenesis regarding $T$. pyogenes.

\section{Methods}

\section{Bacterial culture}

Single colonies of T. pyogenes strains 0912 and 1005 were obtained by inoculating the bacteria on Martin broth agar plates (AOBOX Biotechnology Co., Beijing, China) with $10 \%$ sheep blood. The isolates originated from the lung of cattle that had dies due to pneumonia in China. The agar plates with the inoculum were incubated at $37^{\circ} \mathrm{C}$ for $48 \mathrm{~h}$. For the enrichment culture of the bacteria, single colonies of each $T$. pyogenes strain were inoculated into liquid Martin broth medium (AOBOX Biotechnology Co., Beijing, China) with $10 \%$ fetal bovine serum (FBS) and shaken at $37{ }^{\circ} \mathrm{C}$ for $24 \mathrm{~h}$. The cultures were adjusted to reach an optical density (OD) $600 \mathrm{~nm}$ value of 1.2 using fresh culture medium, and then inoculated into fresh culture medium at a ratio of $2 \%$.

In some of the experiments, the $\mathrm{pH}$ of liquid Martin broth medium was adjusted to different end points, using $\mathrm{HCl}(1 \mathrm{~mol} / \mathrm{L})$ or $\mathrm{NaOH}(1 \mathrm{~mol} / \mathrm{L})$, as indicated in the results.
For the anaerobic culturing of the bacteria, liquid paraffin was used to seal the surface of the liquid Martin broth medium. After inoculation, a 48-h static culture was used for bacterial proliferation. Moreover, the bacteria were cultured in a microaerophilic environment. Briefly, the bacteria were inoculated into the Martin broth agar plates with $10 \%$ sheep blood. The plates were placed in a jar. AnaeroPack-MicroAero (Mitsubishi Gas Chemical Company, Tokyo, Japan) was used to generate a microaerophilic environment. After $24 \mathrm{~h}$, the lawn from the culture was harvested by washing the plates with sterile phosphate buffer saline (PBS).

In most of the above experiments, bacterial growth was determined by measuring the OD $600 \mathrm{~nm}$ values of the cultures. The purity of the cultures was determined by using streak plate method and Gram staining.

\section{Cloning and expression of fimA, fimC, and fimE genes}

The genome of the bacteria was extracted using a Bacterium genome extraction kit (Tiangen Biotech, Beijing, China) according to the manufacturer's instruction. The genes encoding the Fim A, C, and E proteins were amplified and cloned using the primers listed in Table 1 . The genes were inserted into a prokaryotic expression vector, pET-30a (+) (Novagen, Madison, USA) through the corresponding restriction sites. The transformants were sequenced by BGI International using a T7 promoter and a terminator primer. The recombinant plasmids were named pET-30a (+)-fimA, pET-30a (+)-fimC, and pET30a (+)-fimE.

All these recombinant plasmids were transformed into Escherichia coli Rosetta (DE3) ${ }^{\mathrm{TM}}$ competent cells (Novagen, Madison, USA). The positively transformed E. coli were cultured at $37^{\circ} \mathrm{C}$ until they reached an OD $600 \mathrm{~nm}$ value of 0.6-0.8. Recombinant Fim A (rFim A), Fim C (rFim C), and Fim E (rFim E) were expressed by inducing with $1 \mathrm{mmol} / \mathrm{L}$ isopropyl- $\beta$-D-1-thiogalactoside (IPTG) for $4 \mathrm{~h}$ and were then purified using nickel-charged resin (Merck, Shanghai, China). The purified proteins were dialyzed against PBS with $5 \%$ glycerol at $4{ }^{\circ} \mathrm{C}$ for $48 \mathrm{~h}$. The proteins were quantified using Bradford Protein

Table 1 Primers used for gene cloning and plasmid construction

\begin{tabular}{|c|c|c|}
\hline Names of the primers & Nucleotide ( $5^{\prime}$ to $\left.3^{\prime}\right)$ & Restrict sites \\
\hline fimA forward & CGAGCTCATGGGTGCACTGGTGACGGC & Sacl \\
\hline fimA backward & CCCAAGCTTTAGGCATTACGACGACGAGCGAC & Hind III \\
\hline fimC forward & CGCGGATCCGTGTTTACGGTGGTAACC & EcoR I \\
\hline fim C backward & CCCAAGCTTTTAGCTACGACGACGAGCGTG & Hind III \\
\hline fimE forward & CGAGCTCATGAAGCGAAACAAGTTACGTGC & Sacl \\
\hline fime backward & CCCAAGCTTTTAGACGTCCTTGCGTCGGTT & Hind III \\
\hline
\end{tabular}

Restriction enzyme recognition sites are underlined in each sequence and listed in the right column 
Assay Kit (Beyotime, Beijing, China), according to the manufacturer's instructions and stored at $-80{ }^{\circ} \mathrm{C}$ until use. The signal peptides of these proteins were predicted using DNAMAN (LynnonBiosoft, San Ramon, USA).

\section{Preparation of rabbit sera against T. pyogenes, Fim A, Fim C, and Fim E}

T. pyogenes strain 1005 was cultured in $50 \mathrm{~mL}$ Martin broth medium with $10 \%$ FBS. The culture was harvested after being incubated at $37{ }^{\circ} \mathrm{C}$ for $20 \mathrm{~h}$, and then inactivated by treating with $0.5 \%$ formaldehyde at $37{ }^{\circ} \mathrm{C}$ for $72 \mathrm{~h}$. The inactivated culture was centrifuged and the precipitation was resuspended in $20 \mathrm{~mL}$ normal saline $(0.9 \% \mathrm{NaCl} \mathrm{w} / \mathrm{v})$. Aluminum hydroxide gel suspension was prepared as described in a previous study [14]. The inactive $T$. pyogenes culture was mixed with aluminum hydroxide gel suspension at a volume ratio of 5:1.

The protocol of the animal experiment was approved by the Ethics Committee on the Use and Care of Animals, Northeast Agricultural University, China.

Rabbit anti-T. pyogenes strain 1005 serum was prepared through subcutaneous inoculation of a New Zealand rabbit with $1 \mathrm{~mL}$ mixture of inactive T. pyogenes $\left(4.1 \times 10^{9}\right.$ cells) and aluminum hydroxide gel twice with an interval of 15 days. Seven days after the second inoculation, the rabbit was boosted with $1 \mathrm{~mL}$ live T. pyogenes $\left(4.1 \times 10^{9}\right.$ cells). Seven days after the boosting, serum was collected from the rabbit and stored at $-20^{\circ} \mathrm{C}$.

Rabbit anti-rFim A, anti-rFim C, and anti-rFim E sera were prepared by immunizing New Zealand rabbits with the corresponding recombinants. Each recombinant protein was used to immunize one rabbit. Each rabbit received two inoculations at a 2 -week interval. A mixture of $500 \mu \mathrm{g}$ recombinant protein and Freund's complete adjuvant (Sigma-Aldrich, Shanghai, China) was used for the first inoculation, and a mixture of $500 \mu \mathrm{g}$ recombinant protein and Freund's incomplete adjuvant (Sigma-Aldrich, Shanghai, China) was used for the second inoculation. Three days after the second immunization, serum was collected and stored at $-20^{\circ} \mathrm{C}$.

\section{Determination of the reactivity of recombinant proteins to anti-T. pyogenes serum}

The reactivity of the three recombinant proteins to rabbit anti- $T$. pyogenes serum were determined by using western blot assay. Purified rFim A, rFim C, and rFim E (10 $\mu$ g each) were separated via sodium dodecyl sulfate polyacrylamide gel electrophoresis (SDS-PAGE) and were transferred onto nitrocellulose (NC) membranes. The NC membranes were blocked with $10 \%$ skim milk in PBST $(0.05 \% \mathrm{v} / \mathrm{v}$ Tween 20 in PBS) for $1 \mathrm{~h}$ at room temperature. The membranes were then washed with PBST 5 times. The anti-T. pyogenes serum was diluted $(1: 10,000)$ and incubated with the $\mathrm{NC}$ membranes at $4{ }^{\circ} \mathrm{C}$ overnight and washed with PBST five times. Horseradish peroxidase (HRP) conjunct goat anti-rabbit IgG (ZSGB-bio, Beijing, China) was diluted (1:5000) and incubated with the NC membranes for $1 \mathrm{~h}$ at room temperature.

To verify the presence of the three recombinant proteins used for the analysis, a parallel immunoblot assay was performed using anti-histidine tag monoclonal antibodies (1:5000) (ZSGB-bio, Beijing, China) and antimouse HRP conjunct secondary antibodies (1:5000) (ZSGB-bio, Beijing, China) that were incubated with the $\mathrm{NC}$ membranes at $4{ }^{\circ} \mathrm{C}$ overnight and for $1 \mathrm{~h}$ at room temperature.

\section{Determination of the expression of Fim A, Fim C, and Fim E in cultured $T$. pyogenes}

The expression of Fim A, Fim C, and Fim E in cultured T. pyogenes were determined by using western blot assay. Briefly, the T. pyogenes strain 0912 and strain 1005 cultures were harvested, and the collected cultures were centrifuged at $4{ }^{\circ} \mathrm{C}$. The precipitations were washed with sterile PBS twice. Moreover, the bacteria were resuspended in sterile PBS and ultrasonically broken down $(500 \mathrm{~W})$ for $10 \mathrm{~min}(5 \mathrm{~s}$ with intervals of $3 \mathrm{~s})$. The products were centrifuged at $4{ }^{\circ} \mathrm{C}$, and the supernatants were harvested for further analysis. The concentrations of the proteins in supernatants were determined using Bradford Protein Assay Kit (Beyotime, Beijing, China). Afterward, the samples were adjusted using the same concentration. A total of $40 \mu \mathrm{g}$ of proteins in samples were separated through SDS-PAGE. The expression of Fim A, Fim C, and Fim $\mathrm{E}$ in the samples were determined using rabbit antirFim A (1:5000), rFim C (1:2000), and rFim E (1:2000) sera, respectively. The primary antibodies were incubated with the NC membranes at $4{ }^{\circ} \mathrm{C}$ overnight. HRP conjugated goat anti-rabbit IgG (ZSGB-bio, Beijing, China) was diluted (1:5000) and incubated with the NC membrane for $1 \mathrm{~h}$ at room temperature.

\section{Tube agglutination test}

T. pyogenes strains 0912 and 1005 cells were cultured at $37{ }^{\circ} \mathrm{C}$ until the OD $600 \mathrm{~nm}$ value reached 1.75 . The cultures, in volumes of $50 \mathrm{~mL}$, were centrifuged at $4{ }^{\circ} \mathrm{C}$. The supernatants were discarded and the precipitates were washed twice with sterile PBS. The final precipitations were resuspended in $25 \mathrm{~mL}$ sterile normal saline. The bacterial suspensions were used as antigens for the tube agglutination test.

Tube agglutination test was utilized to determine the reactivity of the anti-rFim $\mathrm{A}$, anti-rFim $\mathrm{C}$, and anti-rFim E sera to $T$. pyogenes cells. The twofold serially diluted sera by normal saline $(0.5 \mathrm{~mL})$ was mixed with equal volumes of bacterial suspension. The tubes were incubated 
at $37{ }^{\circ} \mathrm{C}$ for $30 \mathrm{~min}$. Unimmunized rabbit serum was added in every set of the test as controls.

\section{Results}

\section{Cloning fimA, fimE, and fim $C$ genes of $T$. pyogenes}

The fimA, fim $C$, and fimE genes were amplified from genomes of T. pyogenes strains 0912 and 1005. The fimA, fim $C$, and fim $E$ genes from $T$. pyogenes strain 0912 were 1362, 1374, and 1755 base pair (bp), respectively. The fimA and fimC genes of T. pyogenes strain 1005 showed the same length as the genes from strain 0912, whereas three nucleotide (382-384) deletions were observed in the fimE gene from $T$. pyogenes strain 1005 , in comparison with fimE gene from strain 0912. The homologies of fimA, fim $C$, and fimE genes from the two strains were $98.83 \%, 99.04 \%$, and $96.75 \%$, respectively. The homologies of the deduced primary structures of Fim A, Fim C, and Fim E protein from the two strains were $98.01 \%$, 99.43\%, and $96.23 \%$, respectively.

The primary structures of putative Fim A, Fim C, and Fim E proteins of T. pyogenes strain 0912 were analyzed using DNAMAN, which predicted signal peptides with lengths of 22, 27, and 30 amino acids (aa) at the carboxyl terminals of the three proteins, respectively. As reported previously [12], the deduced primary structures of Fim A, Fim $C$ and Fim E proteins possess a cell wall sorting signal (CWSS), which is composed of a LPLTG motif and located approximately 30 aa away from the $C$ terminus of these proteins (amino acid sequences of the three putative proteins of $T$. pyogenes strain 0912 are provided in Additional file 1).

Preparation and identification of $r$ Fim $A, r$ Fim $C$, and $r$ Fim $E$ As the target proteins from the two strains showed relatively high homology, only genes from $T$. pyogenes strain
0912 were expressed in E. coli. The rFim A and rFim C showed a molecular weight of approximately $58 \mathrm{kDa}$, whereas rFim E weighed approximately $72 \mathrm{kDa}$ (Fig. 1a). Furthermore, western blot assay showed that rFim E, but not rFim A and rFim C, reacted with the anti-T. pyogenes serum (Fig. 1b). Results indicated that only anti-Fim $\mathrm{E}$ antibodies were elicited when the rabbit was immunized with T. pyogenes.

\section{Determination of the expression of Fim A, Fim C, and Fim E in cultured T. pyogenes strain 0912}

Trueperella pyogenes strain 0912 was cultured for $4 \mathrm{~h}$. The western blot assay indicated that only Fim E (predicted molecular weight was $56.6 \mathrm{kDa}$, without signal peptide and amino acids behind CWSS) was detected in the bacterium (Fig. 2). The blots for Fim A (predicted molecular weight is $42.2 \mathrm{kDa}$ ) and Fim $\mathrm{C}$ (predicted molecular weight was $41.1 \mathrm{kDa}$ ) detection showed nonspecific reaction bands. However, at the predicted area on the blots, none of the bands showed stronger reactivity than those of the other bands with the corresponding antiserum.

\section{Determination of the effects of different cultural conditions on the expression of Fim A, Fim C, and Fim E in T. pyogenes strains 0912 and 1005}

We initially determined the influence of culture period on the expression of the three proteins. As shown in Fig. 3, we sampled T. pyogenes strains 0912 and 1005 cultures at $4,6,8,10,12,16,20$, and 24 h post-inoculation. The expression of Fim E was observed in $T$. pyogenes strains 0912 and 1005 and was detectable in all samples collected at different time points. The expression of Fim E peaked at $6 \mathrm{~h}$ for strain 0912, and $10 \mathrm{~h}$ for strain 1005.

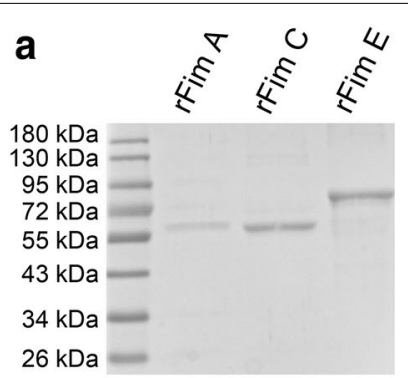

$25 \mathrm{kDa}$

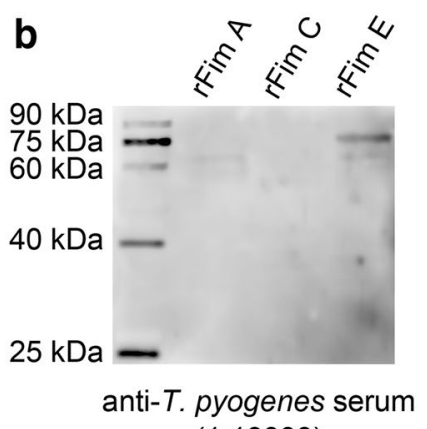

$(1: 10000)$

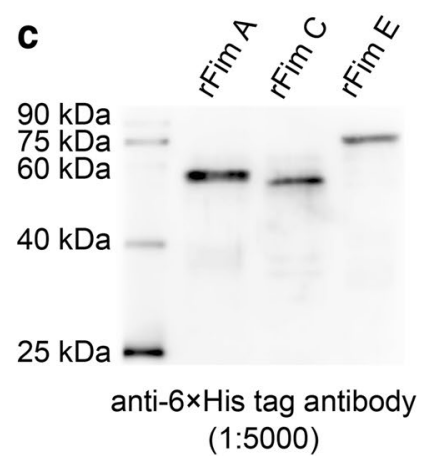

$(1: 5000)$

Fig. 1 Analysis of the molecular weight and immunoreactivity of the rFim A, rFim C, and rFim E proteins by SDS-PAGE and western blot assays. a Purified rFim A, rFim C, and rFim E were analyzed via SDS-PAGE. The predicted molecular weight of rFim A, rFim C, and rFim E was 58 kDa, $58 \mathrm{kDa}$, and $72 \mathrm{kDa}$, respectively. $\mathbf{b}$ Immunoreactivity of rFim A, rFim C, and rFim E to rabbit anti-T. pyogenes serum. The serum was diluted 1:10,000. c Determination of the existence and the amount of rFim A, rFim C, and rFim E on the NC membrane via immunoblot assay using anti-histidine tag monoclonal antibody. The antibody was diluted 1:5000 

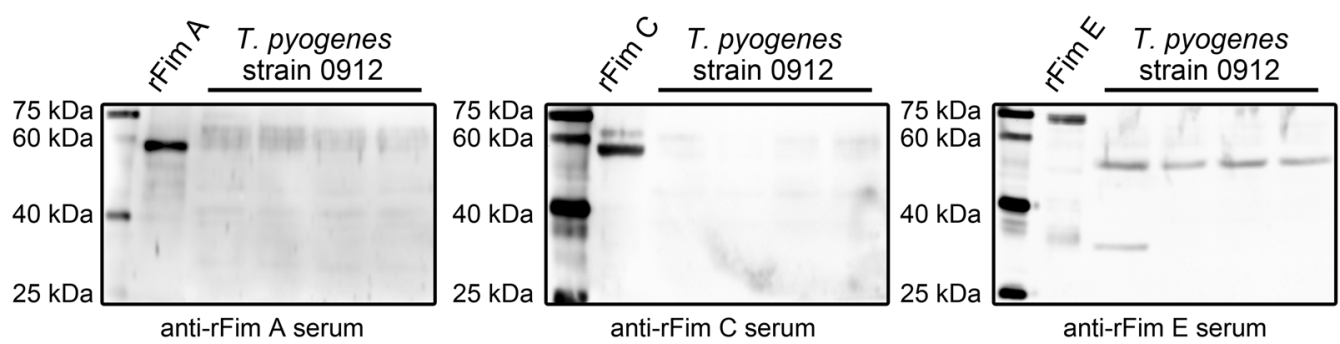

Fig. 2 Analyzing the expression of Fim A, Fim C, and Fim E in cultured T. pyogenes strain 0912 by western blot assays. The samples for immunoblot assay were harvested from four different batches of cultured T. pyogenes strain 0912, all cultured for $4 \mathrm{~h}$

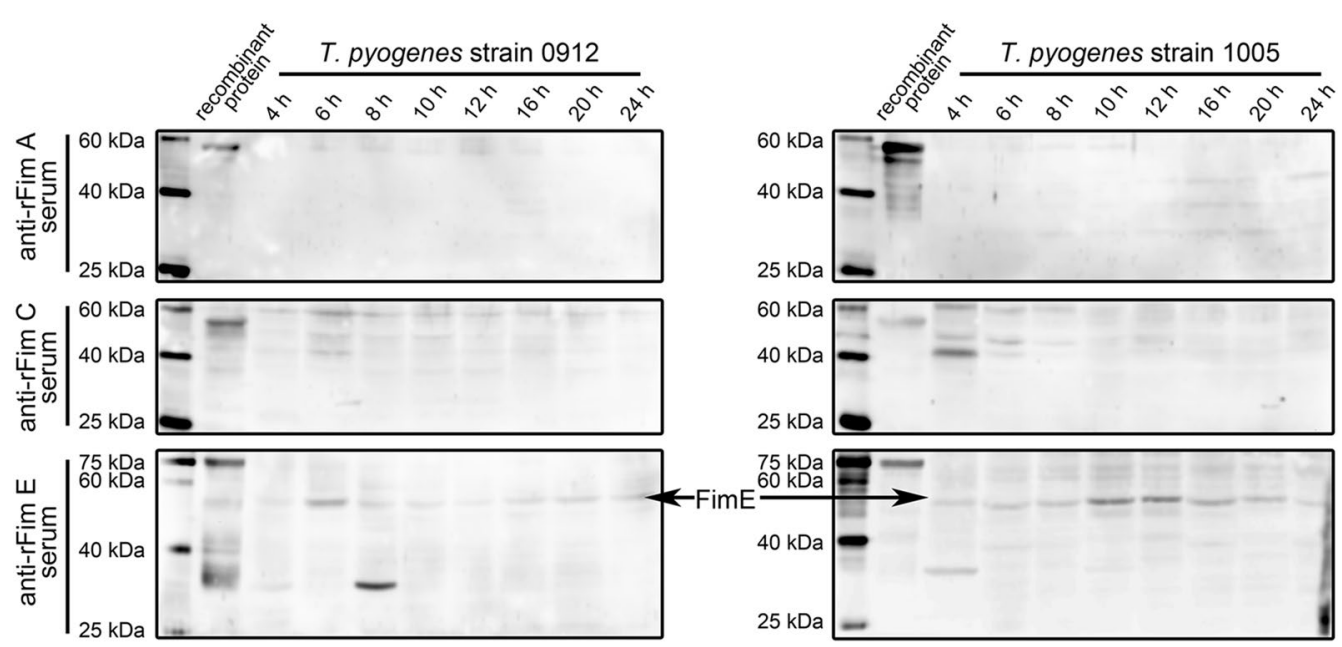

Fig. 3 Analyzing the effect of culture period on the expression of Fim A, Fim C, and Fim E in T. pyogenes strain 0912 and strain 1005 by western blot assays. T. pyogenes strain 0912 and strain 1005 cells were cultured for $24 \mathrm{~h}$. Samples were collected at 4, 6, 8, 10, 12, 16, 20, and 24 h. The experiment was repeated at least three times

The blots for Fim A and Fim C detection showed many nonspecific reaction bands.

We determined the influence of $\mathrm{pH}$ on the expression of the three proteins in T. pyogenes strains 0912 and 1005. Effective proliferation of T. pyogenes was observed at $\mathrm{pH}$ $6.5,7.5$, and 8.5 within $8 \mathrm{~h}$ and were partially inhibited at $\mathrm{pH} 4.5,5.5,9.5$, and 10.5. Figure 4 show that Fim E expression could be observed in organisms cultured at pH 5.5-8.5. The blots for Fim A and Fim $\mathrm{C}$ detection still showed many nonspecific reaction bands.

We finally tried to investigate the influence of anaerobic environment and microaerophilic environment on the expression of the three proteins in T. pyogenes strain 0912. Proliferation of the organism in anaerobic environments was not as efficient as proliferating in aerobic conditions. Figure 5 indicates that anaerobic environment did not affect the expression of the three recombinant proteins, and only the Fim $\mathrm{E}$ was detectable in the western blot assay. The microaerophilic environment improved the growth rate and promoted the production of pyolysin of the microorganisms on the plates (data not shown). However, Fim E was not detected in the microorganism by western blot assay using anti-rFim E serum, thus indicating that the microaerophilic environment could have inhibited the expression of Fim $\mathrm{E}$ (Fig. 5). Moreover, Fim A and Fim C were not detected in T. pyogenes cultured in the microaerophilic environment (Fig. 5).

\section{Tube agglutination test}

Results of tube agglutination test showed that rabbit antirFim C and anti-rFim E sera caused noticeable agglutination of T. pyogenes strains 0912 and 1005 cells at dilution ratios as high as 1:1280 and 1:640 (Fig. 6), respectively. Results indicated that the antigens targeted by the rabbit anti-rFim $\mathrm{C}$ and anti-rFim $\mathrm{E}$ sera were exposed on the surface of the organism.

In contrast, rabbit anti-rFim A serum and the serum collected from an unimmunized rabbit did not cause 


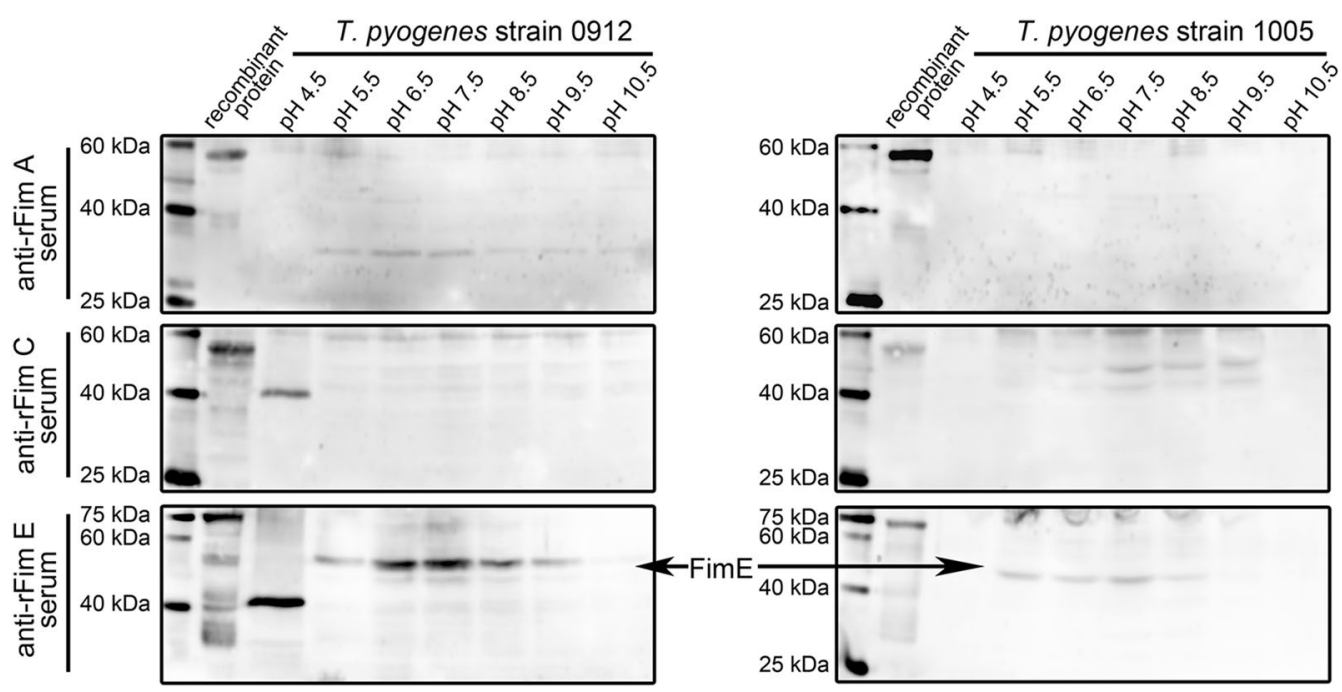

Fig. 4 Analyzing the effect of pH value on the expression of Fim A, Fim C, and Fim E in T. pyogenes strain 0912 and strain 1005 by western blot assays. T. pyogenes strain 0912 and strain 1005 cells were cultured at pH 4.5, 5.5, 6.5, 7.5, 8.5, 9.5, and 10.5 conditions for 8 h. The experiment was repeated at least three times

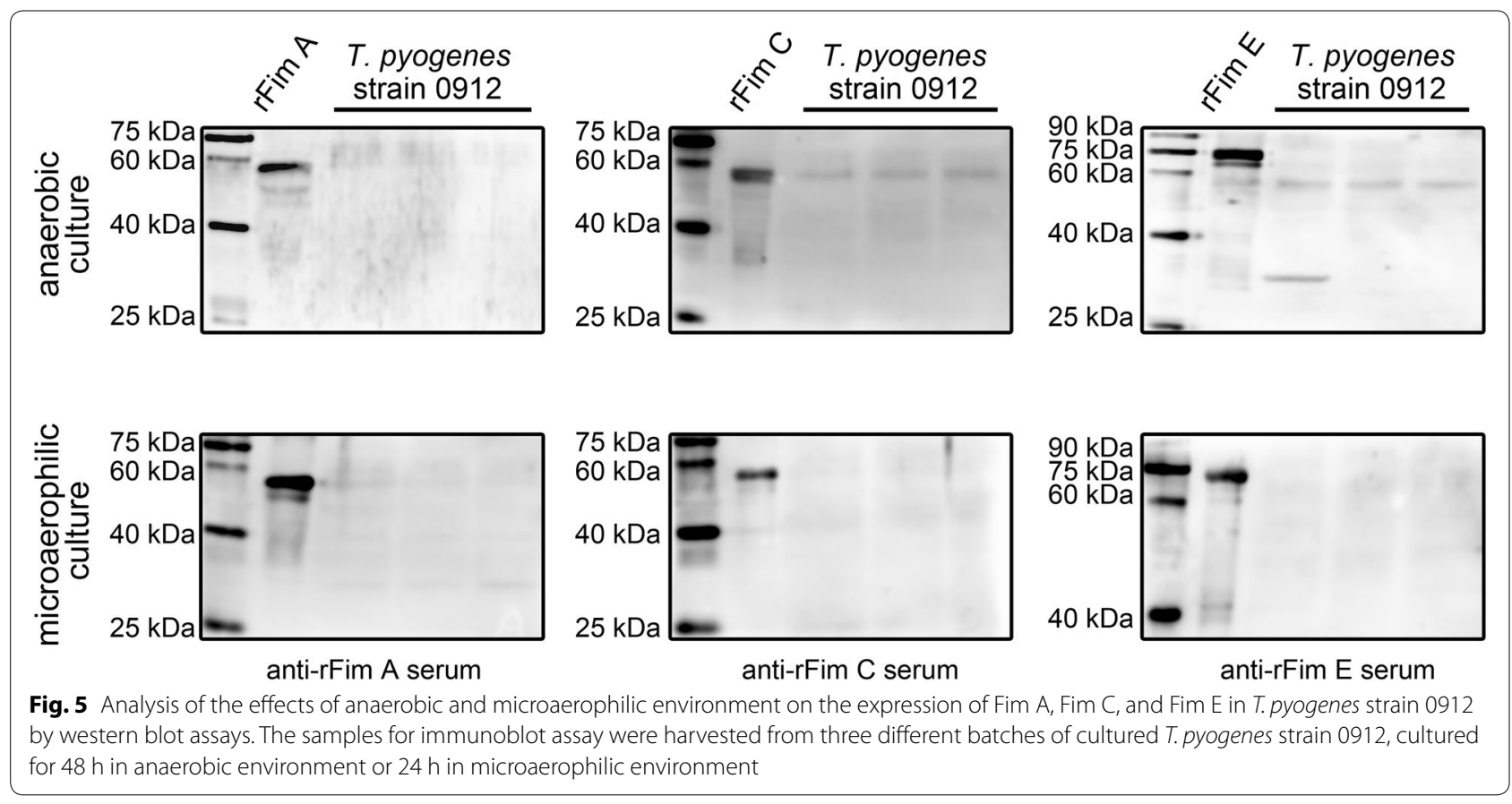

noticeable agglutination of $T$. pyogenes cells within 30 min even at a dilution ratio of as low as 1:40 (Fig. 6).

\section{Discussion}

The pathogenesis of infections caused by the opportunistic pathogen T. pyogenes remain poorly characterized. Opportunistic pathogenic infections take place when the host immune systems are affected by adverse factors, such as shipping, wounds, extreme weather, and primary pathogenic infections, that facilitate the colonization and invasion of these pathogens $[15,16]$. In addition, the changes in expression profiles of the various virulence factors of pathogens might be essential in establishing infections. Jost and Billington [1] speculated that genetically regulated differential expression levels of virulence factors are among the reasons for the transformation of 


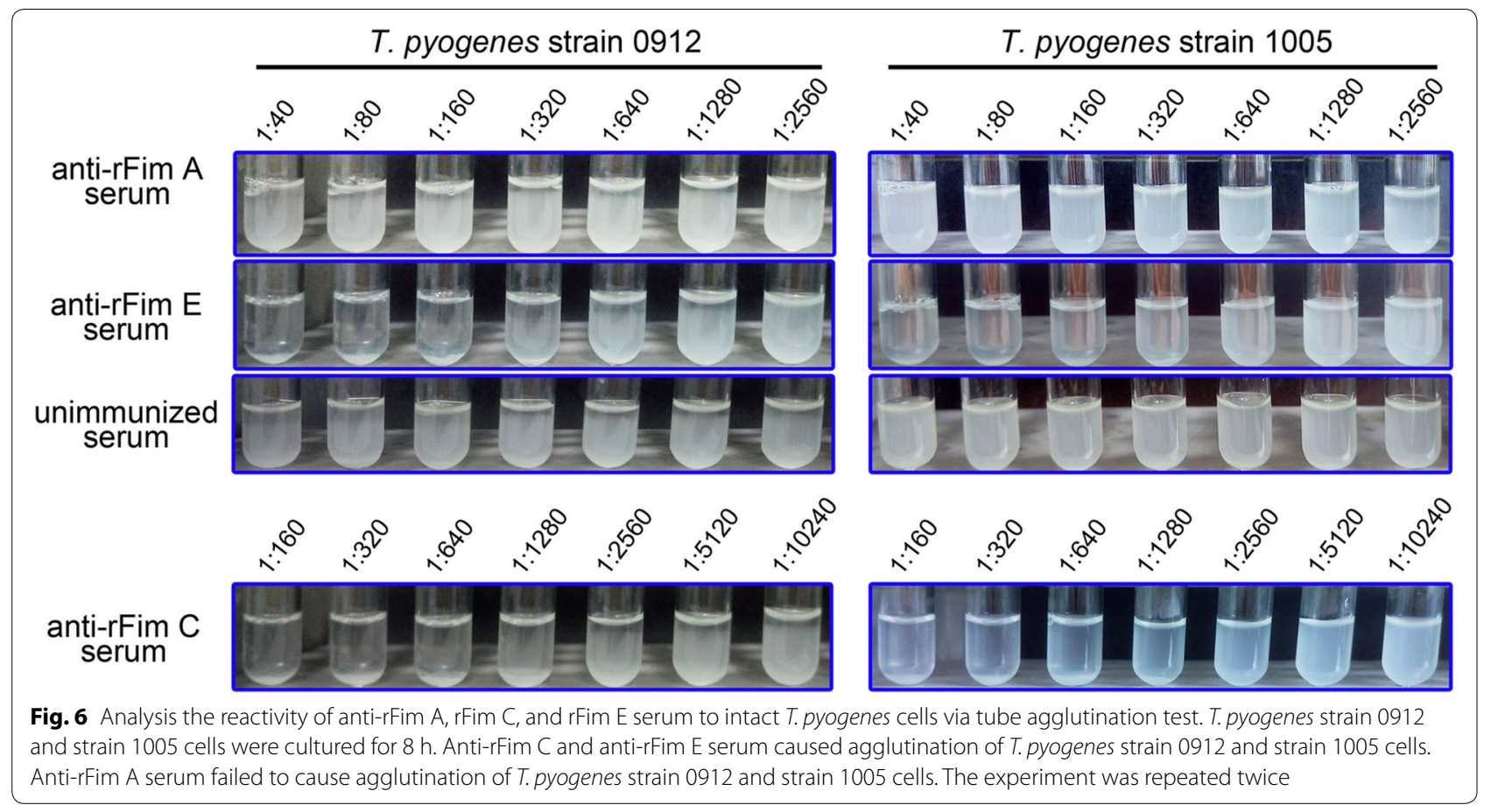

a commensal to a pathogenic organism in T. pyogenes infection. Thus, investigating the expression profiles of the virulence factors of $T$. pyogenes would help in comprehensively understand its pathogenesis. In the current study, the expression of three putative fimbriae proteins (Fim A, Fim C, and Fim E) in cultured T. pyogenes were investigated. The results showed that Fim E protein expression was evident; however, Fim A protein expression was not detected. Moreover, the expression of Fim E was inhibited by microaerophilic conditions.

We first determined the presence of Fim A-, Fim C-, and Fim E-specific antibodies in rabbit anti- $T$. pyogenes serum by immunizing rabbits with the bacterium. The results showed that only rFim $E$ reacted to the anti-serum (Fig. 1), thereby indicating the lack of Fim A- and Fim $\mathrm{C}$-specific antibodies in the serum. Formaldehyde treatment caused the overdenaturation of Fim A and Fim C, which might have caused the phenomenon. However, Fim E molecules in cultured T. pyogenes would also be denatured by the formaldehyde treatment and successfully elicited specific antibodies in rabbit. This implied that the molecules in the T. pyogenes were not overdenatured by the formaldehyde treatment. Additionally, the anti- $T$. pyogenes serum was prepared by immunizing the rabbit with formaldehyde-inactivated and live T. pyogenes. The protocol in the experiment proved the existence of antibodies that were specific to all the components of $T$. pyogenes cells in the serum. Hence, we assumed that the fim $A$ and $f i m C$ genes were either not expressed or expressed in relatively low levels in cultured T. pyogenes, thereby leading to the failure of eliciting specific antibodies against the two proteins.

Then, we directly examined the expression of Fim A, Fim C, and Fim E in cultured T. pyogenes. Results in Figs. 2, 3, 4 indicate that only Fim E could be steadily detected in cultured $T$. pyogenes using western blot assay. The blots for Fim $\mathrm{C}$ detection showed strong nonspecific reaction bands (Figs. 2, 3, 4). Meanwhile, the anti-T. pyogenes serum failed to detect rFim C (Fig. 1). Thus, we could not draw a conclusion related to the expression profile of Fim C in cultured T. pyogenes; however, the anti-rFim $C$ serum caused strong agglutination of T. pyogenes. Bisinotto [12] reported that anti-Fim C serum has been detected in cattle with $T$. pyogenes infection, and that the expression of Fim C by T. pyogenes is observable in vivo. To exactly determine the expression of Fim C in T. pyogenes, specific tools, such as monoclonal antibodies against Fim $C$, are needed. The expression of Fim A was not observed in T. pyogenes lysates using western blot assay (Figs. 2, 3, 4); meanwhile, the antirFim A serum did not cause agglutination in T. pyogenes within $30 \mathrm{~min}$. Several immunoblots showed reactive components and similar molecular weights to the predicted molecular weight of Fim A in T. pyogenes lysates (T. pyogenes strain 1005 sampled at 16, 20, and $24 \mathrm{~h}$ in Fig. 3 and T. pyogenes strain 0912 cultured in pH 5.5 and 6.5 conditions in Fig. 4). Thus, we tested the reactivity of T. pyogenes cultured under these conditions to anti-rFim 
A serum via tube agglutination assay. Results showed no or only weak reaction between $T$. pyogenes and anti-rFim A serum (data not shown). These data indicated the Fim A was not, or poorly, expressed in cultured T. pyogenes in this experiment. The experiment exhibited two limitations: (1) only two isolated strains of T. pyogenes were involved and (2) a standard ATCC strain of T. pyogenes was lacking. Thus, further investigation is needed to determine whether the results of these experiments are universal in $T$. pyogenes strains, using more $T$. pyogenes strains isolated from different animal species and with different types of $T$. pyogenes infections.

Based on the results of the experiments, the microaerophilic condition promoted the proliferation of $T$. pyogenes, but inhibited the expression of Fim $\mathrm{E}$ in vitro. Neisseria meningitidis has been reported to posttranslationally modify one of its pili for the detachment of individual bacterium cells from the colony and colonizing new sites after several rounds of cell division while attached to a host cell [17]. This implies that the bacteria can regulate the expression of adhesins by controlling their attachment and detachment during infections. We speculated that the rapid proliferation of $T$. pyogenes in microaerophilic condition may spread $T$. pyogenes cells, thereby leading to the downregulation of adhesin protein expression. However, in the present study, the microaerophilic culture was only achieved when the organism was cultured on the plate. This led to another speculation that the expression of Fim E in T. pyogenes decreased due to the changes in the culturing method, but not because of the gaseous environment. This hypothesis should be addressed in future studies.

T. pyogenes has been reported to be less efficient to produce fimbriae under standard growth conditions [1]. Yanagawa and Honda [18] reported that only a small portion of cultured T. pyogenes have fimbriae (10\%), and that the number of fimbriae on each piliated bacterial cell is less than 10. On the basis of our results, we speculated that the poor expression of fimbriae was at least partially attributed to the poor expression of Fim A, if the putative Fim A indeed participates in the assembly of $T$. pyogenes fimbriae.

Jost and Billington [1] and Bisinotto [12] reported the existence of antibodies against Fim A in T. pyogenesinfected cattle, thus indicating the expression of Fim A by $T$. pyogenes in vivo. Moreover, Bicalho [19] reported that the increased transcription of fimA gene of $T$. pyogenes is associated with the occurrence of metritis and endometritis in postpartum dairy cows. However, the results of Bisinotto's study inferred the poor expression of Fim A by T. pyogenes compared with the expression levels of Fim $C$ and Fim E, because the OD value of the ELISA for Fim A-specific antibody detection was noticeably lower than those of the ELISA for Fim C and Fim E-specific antibody detection [12].

An interesting phenomenon in Bisinotto's report is that the titers of antibodies against Fim $\mathrm{C}$ and Fim E were associated with the presence of $T$. pyogenes in the uterus of cattle (constantly detectable if the $T$. pyogenes was detected in the cattle and consistently higher than that of herdmates without $T$. pyogenes), but were not significantly related to the day relative to calving. In contrast, the titers of antibodies against Fim A were not associated with the presence of $T$. pyogenes. However, the increase of the concentration of Fim A-specific antibodies was significantly related to the day relative to calving in cows with T. pyogenes [12]. Thus, we speculated that the expression of Fim $\mathrm{C}$ and Fim E by T. pyogenes in vivo are constitutive, whereas the expression of Fim A might be regulated by several factors related to the calving of the cattle.

Another possible mechanism related to the upregulation of Fim A expression in vivo might be a quorumsensing response. Duarte [20] reported that the genome of $T$. pyogenes involves the lsrACDBFGE operon, which is related to the quorum-sensing signal response AI-2. Huang [21] reported that $\mathrm{N}$-acyl homoserine lactones, a type of quorum-sensing molecule, significantly decreased the expression levels of virulence genes of residual $T$. pyogenes in a mouse model. These data infer the importance of quorum sensing in regulating the expression of virulence factors in T. pyogenes. Wagener [22] reported that the proportion of T. pyogenes-positive cows increased from $0.5 \%$ at day $0-85 \%$ at 15 th day postpartum, as determined via traditional bacterial isolation method, thereby indicating the proliferation of $T$. pyogenes in the uterus of the cattle. Moreover, Williams [23] reported that the growth density of $T$. pyogenes from uterine lumen swabs collected from postpartum cows peaked in samples collected at the 14th day postpartum. The proliferation of the organism in the cattle uterus might raise the concentration of quorumsensing molecules in tissues and upregulate the Fim A expression.

Combining the results of the previous and current studies, we speculated that the poor expression of Fim A restricts the formation of additional fimbriae by $T$. pyogenes in normal circumstances, and that $T$. pyogenes shows commensal characteristics. When the environmental conditions changed, the upregulation of Fim A expression promoted fimbriae assembly. Additional fimbriae and the upregulation of other virulence factor expressions facilitated the invasion of $T$. pyogenes into deep tissues. In future studies, genetic technology-mediated overexpression of Fim A protein in T. pyogenes, followed by the counting of fimbriae, might be a practical 
strategy to further study the role of Fim A in T. pyogenes infection.

\section{Conclusions}

We determined the expression profile of the three putative fimbriae proteins, namely, Fim A, Fim C, and Fim $\mathrm{E}$, in in vitro cultured T. pyogenes. Based on our results, only Fim E could be detected via western blot method. The expression of Fim E could be inhibited by microaerophilic condition. Our results would be helpful for comprehensively understanding the pathogenesis of $T$. pyogenes infections and developing T. pyogenes vaccines. The expression profile and role of Fim A and Fim $C$ in $T$. pyogenes should be further studied, using more sensitive and specific tools and proper strategies.

\section{Additional file}

Additional file 1. The amino acid sequences of the putative Fim A, Fim C and Fim E of T. pyogenes strain 0912. The amino acids in CWSS of each protein were colored by red.

\begin{abstract}
Abbreviations
aa: amino acids; bp: base pair; CWSS: cell wall sorting signal; NC: nitrocellulose; FBS: fetal bovine serum; HRP: horse radish peroxidase; IPTG: isopropyl- $\beta$-D1-thiogalactoside; OD: optical density; PBS: phosphate buffer saline; rFim A: recombinant Fim A; rFim C: recombinant Fim C; rFim E: recombinant Fim E; SDS-PAGE: sodium dodecyl sulfate polyacrylamide gel electrophoresis; T. pyogenes: Trueperella pyogenes.
\end{abstract}

\section{Authors' contributions}

WZ designed the experiments, analyzed the results, supervised the work, and drafted the manuscript. ML prepared the recombinant proteins and antisera and performed the western blot assays and tube agglutination tests. BW cloned the genes and $\mathrm{HL}$ provided help to purify the recombinant proteins. BM and JW supervised the work. All authors read and approved the final manuscript.

\section{Author details}

${ }^{1}$ College of Veterinary Medicine, Northeast Agricultural University, Changjiang Road 600, Harbin 150030, Heilongjiang, People's Republic of China. ${ }^{2}$ Key Laboratory of Animal Pathogen Biology of Ministry of Agriculture and Rural Affairs of the People's Republic of China, Northeastern Science Inspection Station Harbin 150030, Heilongjiang, People's Republic of China.

\section{Competing interests}

The authors declare that they have no competing interests.

\section{Availability of data and materials}

The datasets used and/or analysed during the current study are available from the corresponding author on reasonable request.

\section{Consent for publication}

Not applicable.

\section{Ethics approval and consent to participate}

The experimental protocol on animals was approved by the Ethics Committee on the Use and Care of Animals, Northeast Agricultural University on the basis of the Guide for the Care and Use of Laboratory Animals (Institute of Laboratory Animal Resources, Commission on Life Sciences, National Research Council, 2000).

\section{Funding}

This work was supported by National Natural Science Foundation of China (Grant Nos. 31672529 and 31402177), the University Nursing Program for Young Scholars with Creative Talents in Heilongjiang Province (Grant No. UNPYSCT-2017019), the "Academic Backbone" Project of Northeast Agricultural University (Grant No. 17XG09).

\section{Publisher's Note}

Springer Nature remains neutral with regard to jurisdictional claims in published maps and institutional affiliations.

Received: 23 April 2018 Accepted: 9 September 2018

Published online: 12 September 2018

\section{References}

1. Jost BH, Billington SJ. Arcanobacterium pyogenes: molecular pathogenesis of an animal opportunist. Antonie Van Leeuwenhoek. 2005;88:87-102.

2. Nagib S, Glaeser SP, Eisenberg T, Sammra O, Lammler C, Kampfer P, et al. Fatal infection in three Grey Slender Lorises (Loris lydekkerianus nordicus) caused by clonally related Trueperella pyogenes. BMC Vet Res. 2017;13:273.

3. Risseti RM, Zastempowska E, Twaruzek M, Lassa H, Pantoja JCF, de Vargas APC, et al. Virulence markers associated with Trueperella pyogenes infections in livestock and companion animals. Lett Appl Microbiol. 2017;65:125-32.

4. Wickhorst JP, Hassan AA, Sheet OH, Eisenberg T, Sammra O, Alssahen $M$, et al. Trueperella pyogenes isolated from a brain abscess of an adult roebuck (Capreolus capreolus). Folia Microbiol. 2018;63:17-22.

5. Kavitha K, Latha R, Udayashankar C, Jayanthi K, Oudeacoumar P. Three cases of Arcanobacterium pyogenes-associated soft tissue infection. J Med Microbiol. 2010;59:736-9.

6. Patel S, Mathivanan N, Goyal A. Bacterial adhesins, the pathogenic weapons to trick host defense arsenal. Biomed Pharmacother. 2017;93:763-71.

7. Solanki V, Tiwari M, Tiwari V. Host-bacteria interaction and adhesin study for development of therapeutics. Int J Biol Macromol. 2018;112:54-64.

8. Rogovskyy AS, Lawhon S, Kuczmanski K, Gillis DC, Wu J, Hurley H, et al. Phenotypic and genotypic characteristics of Trueperella pyogenes isolated from ruminants. J Vet Diagn Invest. 2018;30:348-53.

9. Esmay PA, Billington SJ, Link MA, Songer JG, Jost BH. The Arcanobacterium pyogenes collagen-binding protein, CbpA, promotes adhesion to host cells. Infect Immun. 2003;71:4368-74.

10. Jost BH, Songer JG, Billington SJ. Cloning, expression, and characterization of a neuraminidase gene from Arcanobacterium pyogenes. Infect Immun. 2001;69:4430-7.

11. Jost BH, Songer JG, Billington SJ. Identification of a second Arcanobacterium pyogenes neuraminidase and involvement of neuraminidase activity in host cell adhesion. Infect Immun. 2002;70:1106-12.

12. Bisinotto RS, Filho JCO, Narbus C, Machado VS, Murray E, Bicalho RC. Identification of fimbrial subunits in the genome of Trueperella pyogenes and association between serum antibodies against fimbrial proteins and uterine conditions in dairy cows. J Dairy Sci. 2016;99:3765-76.

13. Zhao K, Liu M, Zhang X, Wang H, Yue B. In vitro and in vivo expression of virulence genes in Trueperella pyogenes based on a mouse model. Vet Microbiol. 2013:163:344-50.

14. Zhang W, Wang P, Wang B, Ma B, Wang J. A combined Clostridium perfringens/Trueperella pyogenes inactivated vaccine induces complete immunoprotection in a mouse model. Biologicals. 2017;47:1-10.

15. Confer AW. Update on bacterial pathogenesis in BRD. Anim Health Res Rev. 2009;10:145-8.

16. Zhang W, Liu X, Liu M, Ma B, Xu L, Wang J. Development of a multiplex PCR for simultaneous detection of Pasteurella multocida, Mannheimia haemolytica and Trueperella pyogenes. Acta Vet Hung. 2017;65:327-39.

17. Moorthy S, Keklak J, Klein EA. Perspective: adhesion mediated signal transduction in bacterial pathogens. Pathogens. 2016;5:23. https://doi. org/10.3390/pathogens5010023.

18. Yanagawa R, Honda E. Presence of pili in species of human and animal parasites and pathogens of the genus Corynebacterium. Infect Immun. 1976;13:1293-5. 
19. Bicalho ML, Machado VS, Oikonomou G, Gilbert RO, Bicalho RC. Association between virulence factors of Escherichia coli, Fusobacterium necrophorum, and Arcanobacterium pyogenes and uterine diseases of dairy cows. Vet Microbiol. 2012;157:125-31.

20. Duarte VDS, Treu L, Campanaro S, Dias RS, Silva CCD, Giacomini A, et al. The complete genome sequence of Trueperella pyogenes UFV1 reveals a processing system involved in the quorum-sensing signal response. Genome Announc. 2017;5:e0639-717. https://doi.org/10.1128/genom ea.00639-17.
21. Huang $T$, Song $X$, Zhao K, Jing J, Shen $Y$, Zhang $X$, et al. Quorum-sensing molecules $N$-acyl homoserine lactones inhibit Trueperella pyogenes infection in mouse model. Vet Microbiol. 2018;213:89-94.

22. Wagener K, Grunert T, Prunner I, Ehling-Schulz M, Drillich M. Dynamics of uterine infections with Escherichia coli, Streptococcus uberis and Trueperella pyogenes in post-partum dairy cows and their association with clinical endometritis. Vet J. 2014;202:527-32.

23. Williams EJ. Drivers of post-partum uterine disease in dairy cattle. Reprod Domest Anim. 2013:48:53-8.
Ready to submit your research? Choose BMC and benefit from:

- fast, convenient online submission

- thorough peer review by experienced researchers in your field

- rapid publication on acceptance

- support for research data, including large and complex data types

- gold Open Access which fosters wider collaboration and increased citations

- maximum visibility for your research: over $100 \mathrm{M}$ website views per year

At BMC, research is always in progress.

Learn more biomedcentral.com/submissions 